\title{
Tapentadol Prolonged Release for Severe Chronic Osteoarthritis Pain in the Elderly-A Subgroup Analysis of Routine Clinical Practice Data
}

\author{
Andreas Schwittay, ${ }^{1}$ Melanie Sohns, ${ }^{2}$ Birgit Heckes, ${ }^{2}$ and Christian Elling $\mathbb{D D}^{2}$ \\ ${ }^{1}$ Practice for General Medicine, Special Pain Therapy \& Palliative Medicine, Leipziger Str. 2, 04564 Böhlen, Germany \\ ${ }^{2}$ Grünenthal GmbH, Zieglerstr. 6, 52078 Aachen, Germany \\ Correspondence should be addressed to Christian Elling; christian.elling@grunenthal.com
}

Received 18 October 2019; Revised 28 February 2020; Accepted 11 March 2020; Published 14 April 2020

Academic Editor: Massimo Allegri

Copyright (c) 2020 Andreas Schwittay et al. This is an open access article distributed under the Creative Commons Attribution License, which permits unrestricted use, distribution, and reproduction in any medium, provided the original work is properly cited.

\begin{abstract}
Background. Tapentadol prolonged release (PR) has been shown effective and generally well tolerated in a broad range of chronic pain conditions. This subgroup analysis investigated its benefits for elderly patients with severe chronic osteoarthritis (OA) pain in routine clinical practice. Patients and Methods. Data of all patients with chronic OA pain were extracted from the database of a prospective, 3-month noninterventional tapentadol PR trial. The data of elderly OA patients ( $>65$ years of age; $n=752$ ) were compared with the data of younger OA patients ( $\leq 65$ years; $n=282$ ). Results. Almost all patients (elderly $98.7 \%$ and younger patients $99.3 \%$ ) had received long-term analgesic medication prior to the start of tapentadol PR treatment but presented with severe pain accompanied by considerable impairments in sleep quality and quality of life measures. Tapentadol PR provided effective pain relief in both patient groups, with slightly better outcomes in younger patients. However, the mean baseline pain intensity of 7.1 (SD 1.5) was reduced by 3.8 points $(p \leq 0.001)$, and sleep and quality of life measures had also markedly improved in the elderly: quality of sleep by 3 points, quality of life by 3.4 points, social activities by 3 points, and independence by 2.7 points ( $p \leq 0.001$ for all measures; 11-point scale). At the end of observation, $68 \%$ of the elderly had clinically relevant pain reductions of at least $50 \%$ (vs baseline), and $87.9 \%$ attained either their intended pain reduction target and/or an additional individual treatment target (both predefined during baseline examination). Only $8.4 \%$ of the elderly experienced adverse drug reactions, most frequently nausea (2.7\% of patients) and dizziness (1.5\%). Conclusion. Tapentadol PR provided effective and well-tolerated treatment of severe chronic OA pain for elderly patients in routine clinical practice. The favorable tolerability profile in particular suggests tapentadol PR as a treatment option before classical strong opioids are considered.
\end{abstract}

\section{Introduction}

Chronic pain is a common healthcare problem in the general adult population of the European Union and incurs high total population costs [1]. Many chronic pain patients are elderly, and this number is increasing in view of the aging population. Pain treatment is challenging in this age group; pain is often multifactorial and can be more difficult to assess, in particular, when communication and cognitive capacities decline [2]. Furthermore, multiple comorbidities can make the treatment of pain complex.
Tapentadol prolonged release (PR) has been shown to be effective and generally well tolerated in a broad range of chronic pain conditions [3]; trial data of up to 2 years of treatment also indicate good long-term safety and efficacy [4]. An analysis of routine clinical practice data since the introduction of tapentadol confirms the favorable overall safety profile of the medication [5]. In the management of moderate to severe chronic painful osteoarthritis (OA), one of the leading causes of disability in the elderly [6], tapentadol PR provided beneficial effects in several clinical trials $[4,7-13]$ including trials and subgroup analyses in the 
elderly [11-13]. The comparison to oxycodone controlled release (CR) suggested better pain relief and a more improved overall health status under tapentadol PR treatment [14]. The tolerability profile, in particular regarding gastrointestinal side effects, was also more favorable [12-14]. The latter is thought to be a consequence of a reduced $\mu$-opioid load [15] due to the combination of two synergistic mechanisms of action ( $\mu$-opioid receptor agonism and noradrenaline reuptake inhibition) within the tapentadol molecule [16]. To investigate the administration of tapentadol PR in elderly patients under real-world clinical management conditions, we extracted the data of all patients with chronic OA pain from the database of a noninterventional tapentadol PR trial [17] and compared the data of elderly OA patients ( $>65$ years of age) with the data of younger OA patients ( $\leq 65$ years).

\section{Patients and Methods}

The presented data were extracted from the database of a prospective, noninterventional trial conducted between October 2010 and June 2011 in routine clinical practice (general practitioners and internists) in accordance with Section 4, Subsection 23, sentence 3 and Section 67, Subsection 6 of the Medicinal Products Act of the Federal Republic of Germany [17]. As required by law, the German Federal Institute for Drugs and Medical Devices, the German Association of Statutory Health Insurance Physicians, and the German Federal Associations of Health Insurance Funds were notified of the trial. The trial investigated the routine administration of tapentadol PR in clinical practice. Adult patients with severe chronic pain, who-in the opinion of the treating physician-were indicated for tapentadol PR treatment, could be enrolled. All treatment decisions were solely at the discretion of the treating physician. Tapentadol PR was prescribed in accordance with the summary of product characteristics current at the time [18] depending on previous treatment and pain severity. Adjustment of initial tapentadol PR doses within 3 days was recommended in case of insufficient analgesia (maximum recommended daily dose of $500 \mathrm{mg}$ ).

2.1. Data Collection. A total of 1432 medical centers in Germany were involved in data collection in the original trial [17]. Physicians documented data on case report forms during consultation at three time points: baseline examination, treatment follow-up after 4-6 weeks, and at end of observation after approx. 3 months. Documentation included patient demographics, pain diagnosis, previous longterm analgesic treatment, concomitant diseases, the reason for prescribing tapentadol PR, tapentadol dosages, and concomitant analgesics and co-analgesics and adjuvants. Patients were asked about their average pain intensity during the past 3 days, the course of pain over the previous $24 \mathrm{~h}$, and impairments of sleep quality, quality of life, social activities, and independence in the previous 4 weeks. They rated these outcome measures on an 11-point numerical rating scale (NRS-11), with $0=$ no pain to $10=$ worst imaginable pain for pain intensity and $0=$ no impairment to $10=$ maximally conceivable impairment for pain-related impairments. During baseline examination, physician and patient jointly decided on treatment targets to be achieved during the treatment period. Apart from a pain intensity target (NRS11 ), they specified an additional-realistically achievable-individual treatment target from the following areas: quality of life, physical functioning, mental well-being, and others. At the end of observation, this target was rated by the patients using a goal attainment scale ("much better than expected," "better than expected," "as expected," "worse than expected," or "much worse than expected" [19]). Successful tapentadol PR treatment was determined by (a) attainment of at least 50\% reduction in pain intensity, (b) attainment of intended pain intensity, (c) attainment of additional individual treatment target, and (d) attainment of (b) and/or (c) (combined response).

Tolerability was assessed by monitoring the occurrence of adverse drug reactions (ADRs; possibly, probably, or definitely related to the trial medication) during the entire observation period (query during visits and spontaneous reports by the patients). These events were recorded on ADR documentation forms. Physicians did not make explicit causality evaluations. Completing the ADR documentation form implied that physicians assumed a causal relationship between the symptoms and tapentadol treatment; that is, they suspected an ADR.

At the end of treatment, physicians evaluated different aspects of tapentadol PR treatment (analgesia, tolerability, balance between efficacy and tolerability, compliance, quality of life, and overall treatment success), and both patients and physicians rated changes in the general condition of the patient since baseline examination.

2.2. Statistical Analysis. For the original trial, completed case report forms were collected by the sponsor from the participating physicians and forwarded to factum $\mathrm{GmbH}$ (Offenbach, Germany) for data processing (double data entry) using the data management program DMSys (version 5.1., SigmaSoft International, Los Gatos, CA, USA) and data check (for completeness, consistency, and plausibility).

All patients with painful OA of the knee and/or hip participating in the original trial were stratified into an elderly age group ( $>65$ years of age) and a younger age group ( $\leq 65$ years of age). Data extraction and effectiveness analysis were performed by factum $\mathrm{GmbH}$ (Offenbach Germany) using the statistics program SPSS (version 15.0.0; SPSS Inc., Chicago, USA).

Data were analyzed descriptively; percentages throughout the article are based on the total number of patients in the respective group (elderly, $n=752$, or younger patients, $n=282$ ) as denominator, unless otherwise stated. $p$ values for changes in pain intensity, quality of sleep, quality of life, social activities, and independence over the course of the trial were calculated using Wilcoxon matched-pairs signed-rank test. Only patients with data at baseline and final visit were included in calculations for changes in these parameters and in calculations of treatment success criteria. 
ADRs were encoded with the Medical Dictionary for Regulatory Activities (MedDRA) version 20.1.

\section{Results}

3.1. Patient Disposition and Demographics. In total, 1038 patients (33.1\% of the original trial population [effectiveness set $n=3134]$ ) experienced painful OA. Stratification by age resulted in an elderly group ( $>65$ years of age) of 752 patients $(72.4 \%)$ and a younger group ( $\leq 65$ years of age) of 282 patients (27.2\%); information regarding age was not available for 4 patients. Thus, 1034 patients were included in effectiveness and tolerability analyses. Table 1 lists the baseline characteristics of both groups. The majority of the elderly were female; gender was more balanced in the younger patient group. More than 55\% of patients in both groups had experienced pain for more than two years. Many patients with painful knee and/or hip OA also suffered from other pain conditions, most commonly low back pain (LBP; elderly $83.9 \%$ /younger group $83.3 \%$ ). The majority (94.2\%/ $80.1 \%)$ also had concomitant diseases; cardiovascular (79.3\%/46.5\%) and metabolic disorders (41.8\%/39.4\%) were most common. Hospitalization due to pain during the 12 months prior to tapentadol PR treatment was documented for $29.7 \%(223 / 750)$ of the elderly and $23.5 \%(66 / 281)$ of the younger group.

Nearly all patients $(98.7 \%$ [738/748]/99.3\% [278/280]) had already received long-term analgesic treatment prior to the start of tapentadol PR treatment (Figure 1), mainly consisting of several analgesics (Table 2). A higher proportion of elderly patients received transdermal fentanyl and buprenorphine compared with the younger group. Table 3 lists the different dosages of strong opioids used immediately prior to tapentadol initiation. In addition, $34.2 \%$ (256/748) of the elderly and $27.1 \%$ (76/280) of the younger patients took analgesic rescue medication (Table 4). Table 4 also lists the proportion of patients with co-analgesics and adjuvant medications.

3.2. Treatment with Tapentadol $P R$. Main reasons why patients were switched to tapentadol PR were insufficient analgesia (elderly $90 \%$ /younger patients $89.7 \%$ ) and insufficient quality of life $(70.3 \% / 75.4 \% ; n=751 / n=281$; multiple responses permitted). Additional reasons were tolerability issues $(39.4 \% / 35.6 \%)$, insufficient balance between efficacy and tolerability $(31.6 \% / 30.3 \%)$, lack of compliance $(11.9 \% /$ $15 \%)$, and drug interactions with concomitant medication $(7.3 \% / 7.5 \%)$

Mean daily tapentadol PR dosages were comparable between the groups at start of treatment but increased to a greater extent over the observation period in younger patients (Table 5). Three patients (two of them elderly) received higher than recommended daily doses of $500 \mathrm{mg}(600 \mathrm{mg} 2$ patients and $750 \mathrm{mg}$ one patient). The mean treatment duration was $87 \pm 29.6$ days for the elderly $(n=745)$ and $92.3 \pm 28.9$ days for younger patients $(n=272)$. The majority of patients in both groups continued tapentadol PR treatment after the treatment follow-up visit at 4-6 weeks (89.2\%
[667/748]/93.6\% [262/280]) and after the 3-month visit (80.5\% [603/749]/82.1\% [230/280]). Main reasons for treatment discontinuation were unsatisfactory analgesia (8.4\%/6.4\%), the occurrence of ADRs (6.8\%/4.3\%), and no further requirement for analgesic treatment (e.g., surgery; $2.5 \% / 5.7 \% ; n=751 / n=280)$. Nineteen elderly $(2.5 \%)$ and 3 younger patients $(1.1 \%)$ discontinued treatment due to hospitalization. Reasons included surgery (7 patients), fall (1), stroke (1), coronary heart disease (1), chronic fistula (1), ataxia (1), decompensated diabetes mellitus (1), exsiccosis/ refusal to eat (1), and not specified (2). Pain was documented as the reason for hospitalization in four patients (all elderly; colitis was additionally listed for one of them); another elderly patient was hospitalized for periradicular therapy. Only 15 elderly patients (2\%) and 2 younger patients $(0.7 \%)$ discontinued within the first 10 days of tapentadol PR treatment. All cited either the occurrence of ADRs (1.9\%/ $0.7 \%)$ and/or unsatisfactory analgesia $(0.7 \% / 0.4 \%)$ as reason for discontinuation. Initial daily dosing for these patients had been $2 \times 50 \mathrm{mg}$ ( 9 elderly, 2 younger patients), $2 \times 100 \mathrm{mg}$ (5 elderly), and $2 \times 150 \mathrm{mg}$ ( 1 elderly).

Given that tapentadol was only available for use to physicians for a limited time period prior to the start of the trial, the doses used in this trial were conservative. Nevertheless, the intake of additional long-term analgesic medication could be reduced over the observation period (Figure 1). At the start of tapentadol PR treatment, 71.2\%/ $71.5 \%$ of patients received additional long-term analgesics (mainly nonopioids), and $20.3 \%$ in both groups received only tapentadol PR, and $8.5 \% / 8.2 \%$ received tapentadol PR plus rescue medication $(n=750 / n=281)$. At the end of observation, the proportion of patients with tapentadol PR monotherapy had increased to $32.2 \%$ and $36.8 \%$, respectively, and an additional $12.9 \% / 10.7 \%$ received tapentadol PR as only long-term medication plus analgesic rescue medication $(n=712 / n=272)$. Additional long-term analgesics consisted mainly of nonopioids (Figure 1). Intake of rescue medication, antidepressants, antiepileptics, antiemetics, and laxatives had declined at the end of observation compared with data prior to tapentadol PR administration (Table 4).

3.3. Treatment Effects. During the 3-month observation period, mean baseline pain intensity of $7.1 \pm 1.5$ for both groups was reduced by 3.8 points in the elderly ( $p \leq 0.001$; Figure 2(a)) and by 4.2 points in the younger group $(p \leq 0.001$; Figure 2(b)). Pain relief was also observed over the course of $24 \mathrm{~h}$. Fewer elderly patients experienced constant pain with pain attacks (53.1\% before tapentadol PR treatment and $12.5 \%$ at the end of observation), and painfree intervals had markedly increased (from $6.1 \%$ to $51 \%$ of patients; ratings at both visits for 576 patients). The data for the younger age group showed a decrease in constant pain with pain attacks from $51.9 \%$ to $12.7 \%$, and an increase in pain-free intervals from $8 \%$ to $60.9 \%$ (ratings at both visits for 212 patients).

At baseline examination, quality of sleep and all quality of life parameters were markedly impaired in both groups; 
TABLE 1: Baseline characteristics of the trial populations.

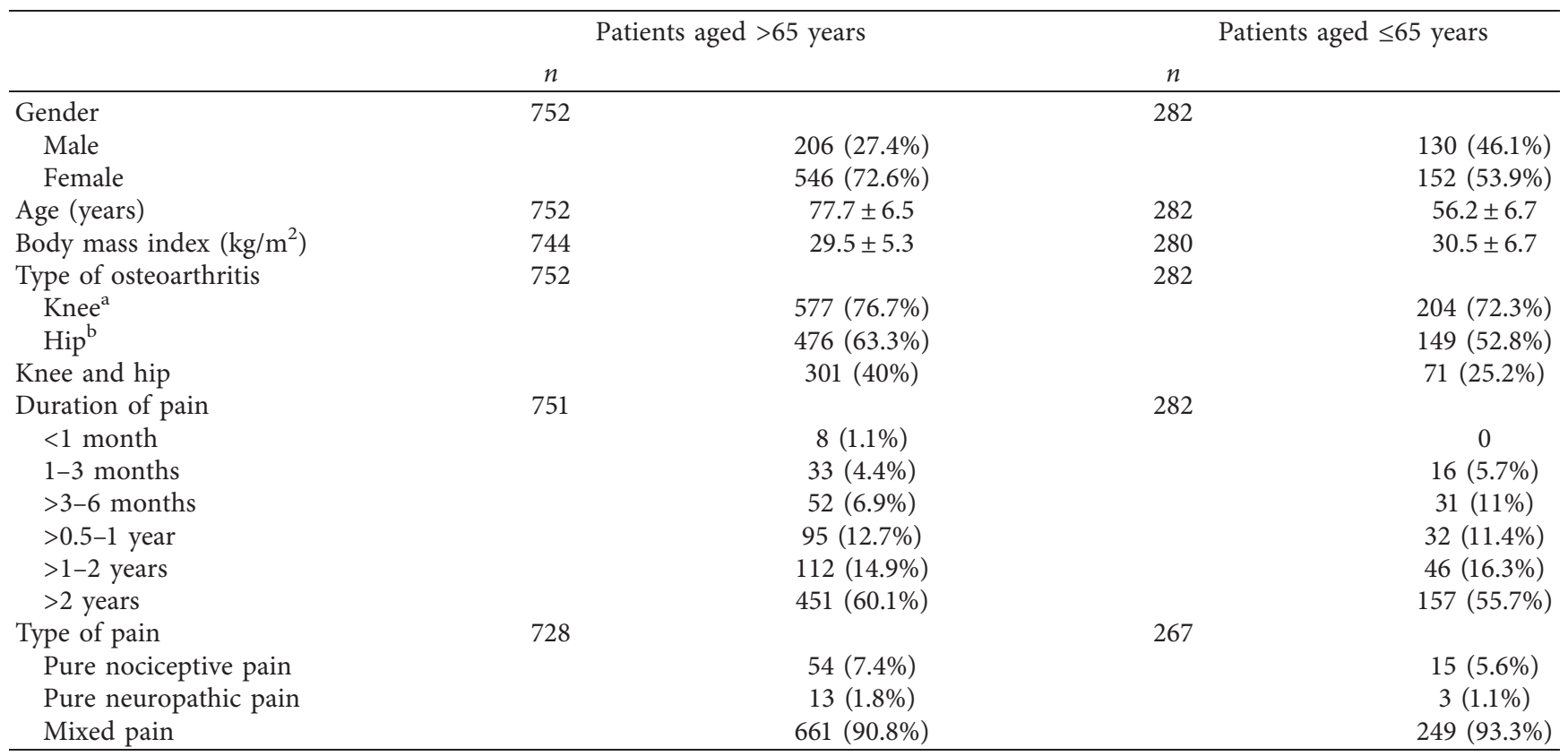

Data are mean \pm SD or number of patients (\%); ${ }^{a}$ all patients with knee osteoarthritis and knee/hip osteoarthritis; ${ }^{b}$ all patients with hip osteoarthritis and hip/ knee osteoarthritis.

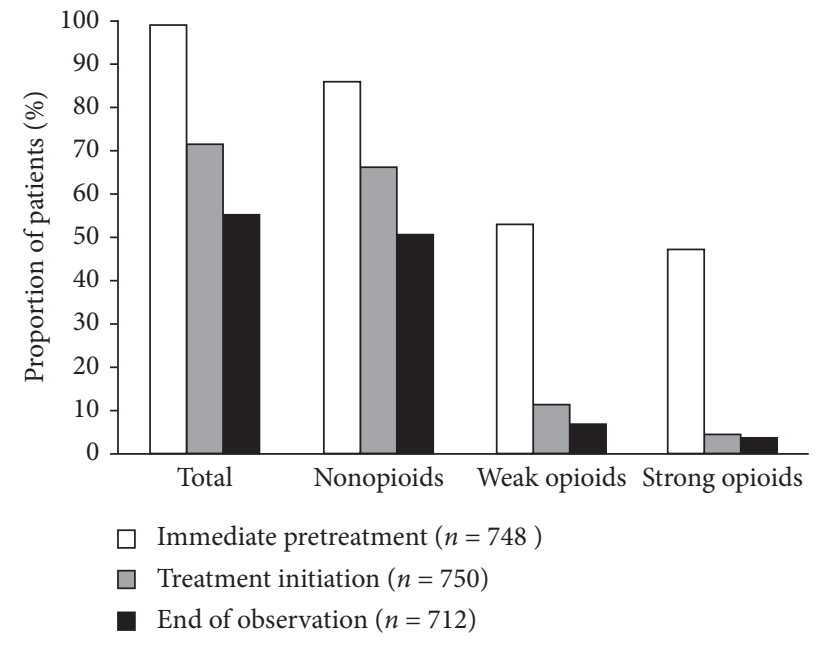

(a)

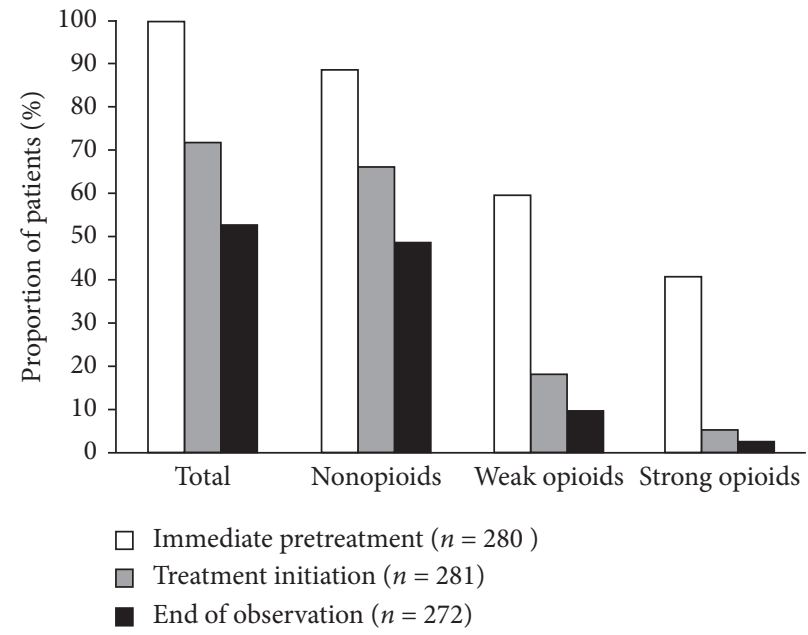

(b)

FIGURE 1: Long-term analgesics immediately preceding initiation of tapentadol PR treatment and change in concomitant analgesics over time. Multiple responses permitted. $n$ number of patients with available data. (a) Osteoarthritis patients $>65$ years of age. (b) Osteoarthritis patients $\leq 65$ years of age.

social activities were affected most (Figure 2). All had considerably improved at the end of observation: quality of sleep by 3 points (elderly) and by 3.6 points (younger patients), quality of life by $3.4 / 3.7$ points, social activities by $3.0 / 3.7$ points, and independence by $2.7 / 2.8$ points (all $p \leq 0.001$; Figure 2). The proportion of patients with uninterrupted sleep increased from $5.9 \%$ to $26.6 \%$ in the elderly (ratings at both visits for 542 patients) and from $4.1 \%$ to $30.3 \%$ in the younger group (ratings at both visits for 218 patients).
3.4. Treatment Success. All four criteria for treatment success were attained by the majority of patients in both groups (Table 6). The mean targeted level of pain intensity at the end of the observation period as defined at baseline was $3.0 \pm 1.2$ by the elderly and $2.7 \pm 1.5$ by the younger group. This target was achieved or exceeded by $65.1 \%(472 / 725) / 65 \%(178 / 274)$ of the patients. The most commonly chosen additional individual treatment targets were from the following areas: quality of life $(73.3 \%[537 / 733] / 73.1 \%$ [198/271]) and physical functioning $(47.2 \% \quad[346 / 733] / 53.5 \% \quad[145 / 271]$; 
TABle 2: Analgesic medication immediately prior to treatment with tapentadol PR.

\begin{tabular}{lcc}
\hline & Aged $>65$ years $(n=752)$ & Aged $\leq 65$ years $(n=282)$ \\
\hline Strong opioids ${ }^{\mathrm{a}}$ & $353(46.9 \%)$ & $113(40.1 \%)$ \\
Fentanyl (transdermal) & $129(17.2 \%)$ & $36(12.8 \%)$ \\
Oxycodone/naloxone & $74(9.8 \%)$ & $30(10.6 \%)$ \\
Oxycodone & $61(8.1 \%)$ & $26(9.2 \%)$ \\
Buprenorphine & $56(7.5 \%)$ & $9(3.2 \%)$ \\
Morphine & $37(4.9 \%)$ & $19(6.7 \%)$ \\
Hydromorphone & $33(4.4 \%)$ & $9(3.2 \%)$ \\
Other & $1(0.1 \%)$ & $1(0.4 \%)$ \\
Weak opioids ${ }^{a}$ & $396(52.7 \%)$ & $166(58.9 \%)$ \\
Tramadol & $214(28.5 \%)$ & $100(35.5 \%)$ \\
Tilidine/naloxone & $200(26.6 \%)$ & $76(27 \%)$ \\
Other/not specified & $1(0.1 \%)$ & 0 \\
Nonopioids ${ }^{\text {a }}$ & $641(85.2 \%)$ & $247(87.6 \%)$ \\
Nonsteroidal anti-inflammatory drugs & $468(62.2 \%)$ & $199(70.6 \%)$ \\
Metamizole & $330(43.9 \%)$ & $105(37.2 \%)$ \\
Muscle relaxants & $115(15.3 \%)$ & $64(22.7 \%)$ \\
Paracetamol & $62(8.2 \%)$ & $34(12.1 \%)$ \\
Other/not specified & $34(4.5 \%)$ & $9(3.2 \%)$ \\
\hline
\end{tabular}

Data are number of patients (\%); ${ }^{a}$ multiple responses permitted. PR, prolonged release.

TABLE 3: Dosages of strong opioids received immediately prior to treatment with tapentadol PR.

\begin{tabular}{ccc}
\hline & Aged $>65$ years & Aged $\leq 65$ years \\
\hline Fentanyl (transdermal) & 129 & 36 \\
$\leq 25 \mu \mathrm{g} / \mathrm{h}$ & 53 & 17 \\
$50 \mu \mathrm{g} / \mathrm{h}$ & 36 & 7 \\
$\geq 75 \mu \mathrm{g} / \mathrm{h}$ & 40 & 12 \\
Oxycodone/naloxone & $74^{\mathrm{a}}$ & 30 \\
$\leq 40 \mathrm{mg} /$ day & 66 & 23 \\
$41-80 \mathrm{mg} /$ day & 7 & 6 \\
$>80 \mathrm{mg} /$ day & 0 & 1 \\
Oxycodone & $61^{\mathrm{a}}$ & 26 \\
$\leq 40 \mathrm{mg} /$ day & 49 & 18 \\
$41-80 \mathrm{mg} /$ day & 8 & 7 \\
$>80 \mathrm{mg} /$ day & 3 & 1 \\
Buprenorphine & $56^{\mathrm{a}}$ & 9 \\
$\leq 20 \mu \mathrm{g} / \mathrm{h}$ & 33 & 9 \\
$35 \mu \mathrm{g} / \mathrm{h}$ & 12 & 0 \\
$52.5 \mu \mathrm{g} / \mathrm{h}$ & 6 & 0 \\
$70 \mu \mathrm{g} / \mathrm{h}$ & 4 & 0 \\
Morphine & 37 & $19^{\mathrm{a}}$ \\
$\leq 60 \mathrm{mg} /$ day & 26 & 10 \\
$61-120 \mathrm{mg} /$ day & 8 & 7 \\
$>120 \mathrm{mg} /$ day & 3 & 1 \\
Hydromorphone & 33 & $9^{\mathrm{a}}$ \\
$\leq 8 \mathrm{mg} /$ day & 8 & 4 \\
$9-16 \mathrm{mg} /$ day & 17 & 2 \\
$>16 \mathrm{mg} /$ day & 8 & 2 \\
\hline & 6 &
\end{tabular}

Data are number of patients. ${ }^{\mathrm{a}}$ Data missing for one patient. PR, prolonged release.

multiple responses possible). More than $85 \%$ of patients in both groups attained their chosen treatment target and the combined response target (Table 6).

3.5. Tolerability. Sixty-three elderly patients (8.4\%) and 18 patients in the younger age group (6.4\%) experienced ADRs during tapentadol PR treatment. The most frequently reported incidences were gastrointestinal and nervous system disorders (Table 7). "Drug withdrawal syndrome" or "withdrawal syndrome" was noted for 4 patients, two of them elderly. The three patients receiving daily tapentadol PR doses over $500 \mathrm{mg}$ did not experience ADRs.

Serious ADRs were reported for 8 elderly patients $(1.1 \%$; 15 ADRs) and 2 younger patients (0.7\%; 3 ADRs). In the elderly group, three patients had one ADR (fall, drug ineffective, and severe cardiac failure), four patients had two ADRs (severe diarrhea + severe sepsis, moderate abdominal pain upper + moderate nausea, ataxia + withdrawal syndrome, and moderate abdominal pain + colitis), and one patient had four moderate ADRs (abnormal sensation in eye, eye movement disorder, vision blurred, and visual impairment). In the younger age group, drug withdrawal syndrome was documented for one patient and severe fecalo$\mathrm{ma}+$ severe ileus paralytic for a second patient.

3.6. Evaluation of Treatment. At the end of observation, tapentadol PR treatment was rated "good" or "very good" for the majority of patients (Table 8). Physicians found the general condition of their patients much or very much improved in $75.2 \%$ [533/709] of elderly patients and in $79.7 \%$ [212/266] of younger patients. Patients' ratings were slightly less positive $(69.1 \%$ [476/689] elderly and $75.9 \%$ [195/257] younger group).

\section{Discussion}

The elderly OA patients analyzed here not only presented with painful knee and/or hip OA but also were afflicted by other pain conditions, most notably LBP. Many had been suffering from pain for more than 2 years, and almost all had received long-term analgesic medication before the start of tapentadol PR treatment. Despite this use of, often strong, analgesics $(47 \%$ of patients were administered WHO III medication), the burden of pain was high: mean pain 
TABle 4: Administration of co-analgesics and adjuvants.

\begin{tabular}{lccc}
\hline & Immediately prior to tapentadol treatment & At the start of tapentadol treatment & At the end of observation \\
\hline Patients aged $>65$ years $(n=752)$ & & & \\
Co-analgesics & $299(39.8 \%)$ & $275(36.6 \%)$ & $249(33.1 \%)$ \\
Antidepressants & $229(30.5 \%)$ & $219(29.1 \%)$ & $205(27.3 \%)$ \\
Antiepileptics & $121(16.1 \%)$ & $97(12.9 \%)$ & $79(10.5 \%)$ \\
No data & 0 & 0 & $30(4 \%)$ \\
Adjuvants & $221(29.4 \%)$ & $197(26.2 \%)$ & $152(20.2 \%)$ \\
Antiemetics & $83(11 \%)$ & $70(9.3 \%)$ & $49(6.5 \%)$ \\
Laxatives & $170(22.6 \%)$ & $144(19.2 \%)$ & $120(16 \%)$ \\
Adjuvants NOS & $1(0.1 \%)$ & $2(0.3 \%)$ & 0 \\
No data & $3(0.4 \%)$ & $3(0.4 \%)$ & $38(5.1 \%)$ \\
Rescue medication & $256(34 \%)$ & $235(31.3 \%)$ & $212(28.2 \%)$ \\
No data & $4(0.5 \%)$ & $2(0.3 \%)$ & $39(5.2 \%)$ \\
Patients aged $\leq 65$ years $(n=282)$ & & & \\
Co-analgesics & $133(47.2 \%)$ & $110(39 \%)$ & $92(32.6 \%)$ \\
Antidepressants & $111(39.4 \%)$ & $99(35.1 \%)$ & $81(28.7 \%)$ \\
Antiepileptics & $54(19.2 \%)$ & $36(12.8 \%)$ & $30(10.6 \%)$ \\
No data & 0 & 0 & $8(2.8 \%)$ \\
Adjuvants & $67(23.8 \%)$ & $53(18.8 \%)$ & $28(9.9 \%)$ \\
Antiemetics & $36(12.8 \%)$ & $28(9.9 \%)$ & $15(5.3 \%)$ \\
Laxatives & $45(16 \%)$ & $33(11.7 \%)$ & $20(7.1 \%)$ \\
Adjuvants NOS & $1(0.4 \%)$ & $1(0.4 \%)$ & 0 \\
No data & $1(0.4 \%)$ & $2(0.7 \%)$ & $13(4.6 \%)$ \\
Rescue medication & $76(27 \%)$ & $14(26.2 \%)$ & $70(24.8 \%)$ \\
No data & $2(0.6 \%)$ & $1(0.4 \%)$ & $10(3.6 \%)$ \\
\hline Nota & &
\end{tabular}

NOS, not otherwise specified. Data are number of patients (\%).

TABLe 5: Daily tapentadol PR doses over the observation period.

\begin{tabular}{lcc}
\hline & Aged $>65$ years $(n=752)$ & Aged $\leq 65$ years $(n=282)$ \\
\hline At the start of treatment (mg) & $129.8 \pm 62.0$ & $132.5 \pm 64.7$ \\
$2 \times 50 \mathrm{mg}$ & $564(75 \%)$ & $204(72.3 \%)$ \\
$2 \times 100 \mathrm{mg}$ & $146(19.4 \%)$ & $57(20.2 \%)$ \\
$\geq 2 \times 150 \mathrm{mg}$ & $33(4.4 \%)$ & $16(5.7 \%)$ \\
Other & $9(1.2 \%)$ & $5(1.8 \%)$ \\
At the end of titration (mg) & $181.5 \pm 92.5$ & $195.1 \pm 93.3$ \\
No data & $12(1.6 \%)$ & $4(1.4 \%)$ \\
At the end of observation (mg) & $198.6 \pm 100.7$ & $213.4 \pm 103.4$ \\
No data & $16(2.1 \%)$ & $5(1.8 \%)$ \\
\hline
\end{tabular}

Data are mean \pm SD or number of patients (\%). PR, prolonged release.

intensity at baseline was rated as severe and accompanied by considerable impairments in sleep quality and daily functioning, and thus markedly reduced quality of life. Not surprisingly, the main reasons physicians opted for a switch to tapentadol PR were insufficient analgesia and quality of life with previous analgesic treatment.

The 3-month treatment with tapentadol PR provided effective pain relief in both patient groups with slightly better outcomes in younger patients. However, a total of $68 \%$ of the elderly patients had clinically relevant pain reductions of at least 50\% (vs baseline), and 65\% achieved their intended pain intensity target. Mean pain intensity at the end of observation deviated by only 0.4 points from the mean treatment target of 3.0 points defined at baseline visit. The results are comparable to findings for the original trial population $(72 \% / 66 \%$ [17]) and the large population of a second 3-month noninterventional tapentadol PR trial (65\%/60\% [20]).
Nausea, dizziness, and constipation were most frequently reported by the $8.4 \%$ of elderly patients experiencing ADRs during tapentadol PR treatment. Only 6.8\% discontinued treatment due to the occurrence of an ADR. Physicians considered tapentadol PR treatment well tolerated by the majority of elderly patients, with "good" to "very good" ratings of over $80 \%$ in the following categories: gastrointestinal tolerability, CNS tolerability, and general tolerability.

Patients suffering from severe pain frequently complain about impairments in daily life such as diminished sleep quality, restrictions in social activities, and loss of independence. These impairments may lead to social isolation, anxiety, and depression, and may thus greatly affect their quality of life. Results for Germany from the 2010 National Health and Wellness Survey showed that the impact of severe and frequent pain on the quality of life is greater than the contribution of sociodemographic factors, the presence 


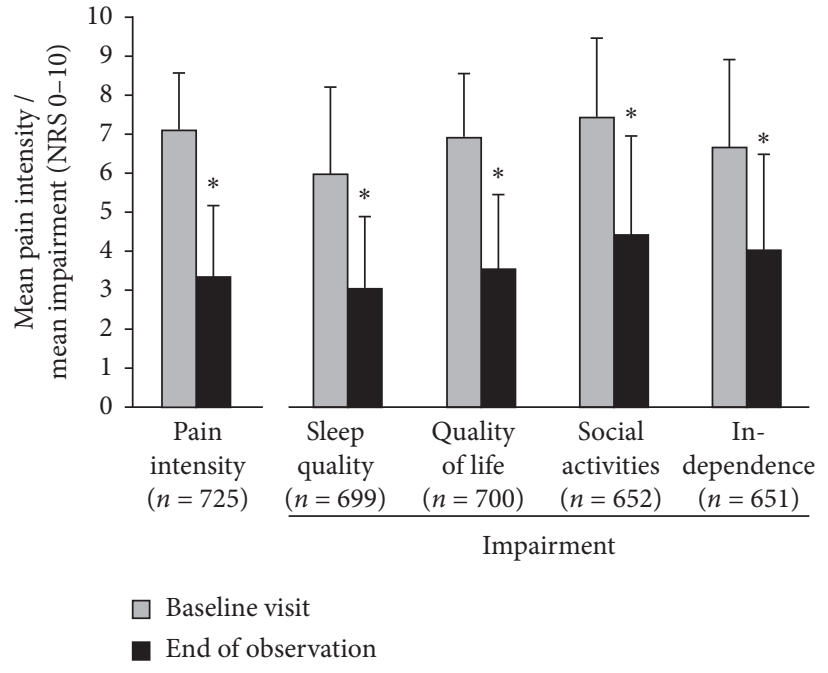

(a)

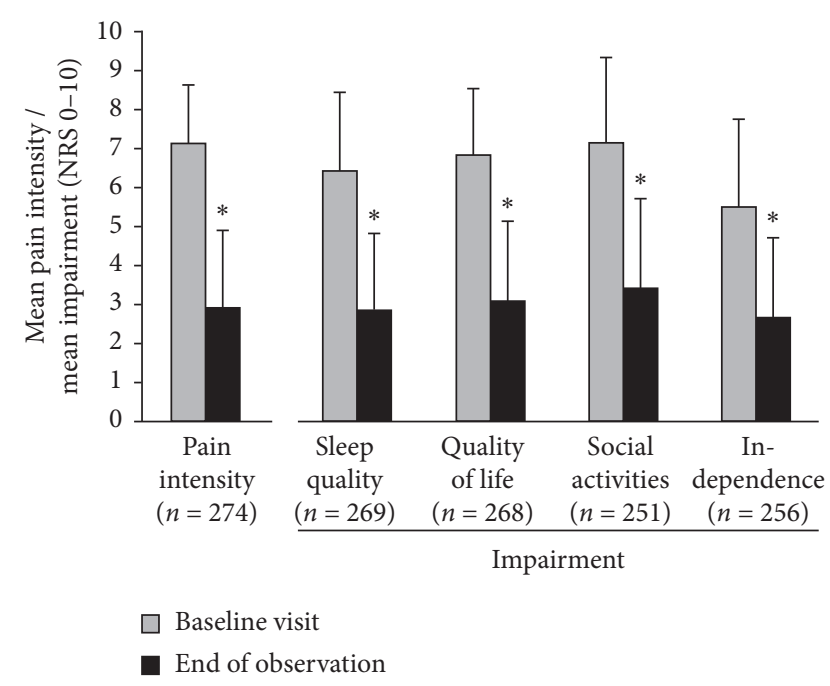

(b)

FIGURE 2: Changes in pain intensity and pain-related impairments of quality of sleep and quality of life parameters (+SD) over the observation period. Only patients with data for both observation time points were included. ${ }^{*} p \leq 0.001$ compared with baseline visit (Wilcoxon matched-pairs signed-rank test). NRS, numerical rating scale. (a) Osteoarthritis patients $>65$ years of age. (b) Osteoarthritis patients $\leq 65$ years of age.

TABLE 6: Proportion of patients who had attained one or several treatment targets at the end of the 3-month tapentadol PR treatment.

\begin{tabular}{lcc}
\hline & Patients aged >65 years & Patients aged $\leq 65$ years \\
\hline (a) Attainment of at least 50\% reduction in pain intensity vs. baseline & $67.6 \%(n=725)$ & $74.1 \%(n=274)$ \\
(b) Attainment of intended pain intensity & $65.1 \%(n=725)$ & $65 \%(n=274)$ \\
(c) Attainment of additional individual treatment target & $87.8 \%(n=713)$ & $91.5 \%(n=272)$ \\
(d) Attainment of (b) and/or (c) (combined response rate) & $87.9 \%(n=728)$ & $92 \%(n=274)$ \\
\hline
\end{tabular}

$n$, number of patients with valid data; PR, prolonged release.

TABLE 7: Most frequently reported adverse drug reactions (incidences of $>1 \%$ of patients for each preferred term).

\begin{tabular}{lcr}
\hline & Patients aged $>65$ years $(n=752)$ & Patients aged $\leq 65$ years $(n=282)$ \\
\hline Any adverse drug reaction & $63(8.4 \%)$ & $18(6.4 \%)$ \\
Gastrointestinal disorders & $33(4.4 \%)$ & $12(4.3 \%)$ \\
Nausea & $20(2.7 \%)$ & $8(2.8 \%)$ \\
Constipation & $8(1.1 \%)$ & $1(0.4 \%)$ \\
Nervous system disorders & $24(3.2 \%)$ & $8(2.8 \%)$ \\
Dizziness & $11(1.5 \%)$ & $6(2.1 \%)$ \\
\hline
\end{tabular}

Data are number of patients (\%).

TABLE 8: Evaluation of tapentadol PR treatment by the treating physicians at the end of observation (proportion of patients with "good" or "very good" ratings).

\begin{tabular}{lcc}
\hline & Patients aged $>65$ years $(n=752)$ & Patients aged $\leq 65$ years $(n=282)$ \\
\hline Analgesia & $76.4 \%(n=698)$ & $85.2 \%(n=270)$ \\
General tolerability & $84.9 \%(n=697)$ & $89.7 \%(n=272)$ \\
Gastrointestinal tolerability & $85.9 \%(n=695)$ & $88.5 \%(n=269)$ \\
CNS tolerability & $86.5 \%(n=691)$ & $91.5 \%(n=271)$ \\
Balance between efficacy and tolerability & $82.5 \%(n=690)$ & $89.5 \%(n=267)$ \\
Quality of life & $70.8 \%(n=694)$ & $81.7 \%(n=268)$ \\
Compliance & $86.9 \%(n=694)$ & $89.5 \%(n=266)$ \\
Overall treatment success & $78.9 \%(n=693)$ & $85.6 \%(n=270)$ \\
\hline
\end{tabular}

$n=$ number of patients with valid data. CNS, central nervous system. PR, prolonged release. 
of comorbidities, or health risk factors such as obesity, alcohol, and smoking [21]. OA pain limits functionality. Physical activity is often restricted, which is considered a major factor in the onset of frailty in the elderly [22], resulting in a loss of independence and ultimately a move to a care facility. Thus, more so than in any other age group, pain relief in the elderly may, for some patients, be key to a longer independent and involved life. In addition, it is also likely to have an impact on the burden of care for their families and costs arising for society in general. These impairments of functionality were observed in our elderly OA patients at baseline. They presented with considerable impairments in sleep quality and quality of life at baseline, with social activities being the most severely impacted quality of life measure. Not surprisingly, the majority considered improvements in quality of life an important treatment target: $73 \%$ of the patients chose an additional individual treatment target from this domain. Effective pain relief under tapentadol PR treatment was accompanied by marked improvements in all quality of life measures which contributed to the overall treatment success of the medication. It should also be noted that one-third of the elderly patients $(32.2 \%)$ did not require additional long-term analgesic medication at the end of observation and a further $12.9 \%$ received only tapentadol $\mathrm{PR}$ plus analgesic rescue medication.

Elderly OA patients received mean daily tapentadol PR doses of $199 \pm 101 \mathrm{mg}$, on average $14 \mathrm{mg}$ less than the younger age group $(213 \pm 103 \mathrm{mg})$ at the end of observation and less than half the maximum recommended daily dose of $500 \mathrm{mg}$. This dosage was at the lower end of the mean daily dose range observed for other routine clinical practice tapentadol PR treatment data in cancer and noncancer pain (192-287 mg [17, 20, 23-27]). In contrast, mean daily doses in phase 3 clinical trials were approx. $300 \mathrm{mg}$ [3] and thus above the higher range found in noninterventional trials. Most of the clinical practice trials had been initiated when tapentadol PR was newly authorized and physicians had not yet gained experience with dosing this new analgesic. They might thus have relied more on additional analgesics instead of using the full tapentadol PR dosing range. Adherence to dose adjustment recommendations available today might further improve therapeutic outcomes including a reduction in the requirement for additional long-term analgesics, an advantage for patients with polypharmacy.

Overall, tapentadol PR was effective and well tolerated in the treatment of painful OA in the elderly in routine clinical practice. The findings complement the positive results of tapentadol PR treatment in elderly patients $\geq 65$ years and $\geq 75$ years of age obtained in pooled analyses of randomized controlled clinical trials $[12,13]$.

Analyses of routine clinical practice data are limited by the absence of a control group. However, our data which derived from a prospective, noninterventional trial where treatment decisions were solely at the discretion of the treating physician provide an insight into real-world pain management and complement the findings of randomized controlled trials with predefined trial criteria. For example, in contrast to randomized controlled trials with balanced group sizes, the proportion of elderly patients in our analysis was much higher (reflecting the real-life situation). However, this did not present a problem as no direct statistical comparison between groups was presented and the large number of patients in both groups allowed for a meaningful analysis within both. The lack in balance of potential confounding factors such as gender (higher proportion of women in elderly compared with younger age group) represents a limitation of our analysis, as this makes the groups not directly comparable to each other and might be reflected in the results of the two groups. However, the focus of our analysis was on the effectiveness and safety of tapentadol in elderly patients in a real-life situation in contrast to what is observed in younger patients in real-life rather than a direct comparison of tapentadol PR efficacy between both groups. It should be noted that most patients in this analysis not only suffered from painful OA but also from LBP. Data analysis could not distinguish between the two pathologies. The coexistence of these two pathologies is often observed in clinical practice and is not surprising, given that one could be caused by the other, for example, insufficient pain treatment of OA might lead to issues with posture resulting in subsequent complaints in other body regions such as the lower back.

\section{Conclusion}

Tapentadol PR provided effective and well-tolerated treatment of severe chronic OA pain for elderly patients in routine clinical practice. The marked improvements in all quality of life measures and the favorable tolerability profile in particular suggest tapentadol PR as an early treatment option before classical strong opioids are considered.

\section{Abbreviations \\ ADR: Adverse drug reaction \\ CR: Controlled release \\ LBP: Low back pain \\ NRS: Numerical rating scale \\ OA: Osteoarthritis \\ PR: $\quad$ Prolonged release \\ WHO: World Health Organization.}

\section{Data Availability}

On a case-by-case basis, the authors will share on request tables of the subgroup analysis.

\section{Conflicts of Interest}

AS is a consultant for Grünenthal $\mathrm{GmbH}$, Germany; he participated in the study and received financial recompense for complete documentation of the patients. MS, BH, and CE are employees of Grünenthal $\mathrm{GmbH}$.

\section{Acknowledgments}

The authors thank all patients and investigators involved in the original trial and Jérôme Rapion (Grünenthal $\mathrm{GmbH}$, 
Germany) for the statistical tolerability analysis. The original trial was funded by Grünenthal $\mathrm{GmbH}$. Extraction and analysis of effectiveness data were performed by factum $\mathrm{GmbH}$ (Offenbach Germany) and were paid for by Grünenthal GmbH. Writing and editorial assistance was provided by Elke Grosselindemann and Birgit Brett and was paid for by Grünenthal $\mathrm{GmbH}$. The sponsor was also involved in the decision to publish.

\section{References}

[1] R. M. Leadley, N. Armstrong, Y. C. Lee, A. Allen, and J. Kleijnen, "Chronic diseases in the European Union: the prevalence and health cost implications of chronic pain," Journal of Pain \& Palliative Care Pharmacotherapy, vol. 26, no. 4, pp. 310-325, 2012.

[2] T. A. Cavalieri, "Pain management in the elderly," Journal of the American Osteopathic Association, vol. 102, pp. 481-485, 2002.

[3] R. Baron, L. Eberhart, K. U. Kern et al., “Tapentadol prolonged release for chronic pain: a review of clinical trials and 5 years of routine clinical practice data," Pain Practice, vol. 17, no. 5, pp. 678-700, 2017.

[4] R. Buynak, S. A. Rappaport, K. Rod et al., "Long-term safety and efficacy of tapentadol extended release following up to 2 years of treatment in patients with moderate to severe, chronic pain: results of an open-label extension trial," Clinical Therapeutics, vol. 37, pp. 2420-2438, 2015.

[5] A. Stollenwerk, M. Sohns, F. Heisig, C. Elling, and D. von Zabern, "Review of post-marketing safety data on tapentadol, a centrally acting analgesic," Advances in Therapy, vol. 35, no. 1, pp. 12-30, 2018.

[6] R. Wittenauer, L. Smith, and K. Aden, Update on 2004 Background Paper, BP 6.12 Osteoarthritis, World Health Organization, Geneva, Switzerland, 2013, http://www.who. int/medicines/areas/priority_medicines/BP6_12Osteo.pdf.

[7] M. Afilalo, M. S. Etropolski, B. Kuperwasser et al., "Efficacy and safety of tapentadol extended release compared with oxycodone controlled release for the management of moderate to severe chronic pain related to osteoarthritis of the knee," Clinical Drug Investigation, vol. 30, no. 8, pp. 489-505, 2010.

[8] J. E. Wild, S. Grond, B. Kuperwasser et al., "Long-term safety and tolerability of tapentadol extended release for the management of chronic low back pain or osteoarthritis pain," Pain Practice, vol. 10, no. 5, pp. 416-427, 2010.

[9] I. Steigerwald, M. Müller, J. Kujawa, J. C. Balblanc, and J. Calvo-Alén, "Effectiveness and safety of tapentadol prolonged release with tapentadol immediate release on-demand for the management of severe, chronic osteoarthritis-related knee pain: results of an open-label, phase 3b study," Journal of Pain Research, vol. 5, pp. 121-138, 2012.

[10] I. Steigerwald, M. Schenk, U. Lahne, P. Gebuhr, D. Falke, and B. Hoggart, "Effectiveness and tolerability of tapentadol prolonged release compared with prior opioid therapy for the management of severe, chronic osteoarthritis pain," Clinical Drug Investigation, vol. 33, no. 9, pp. 607-619, 2013.

[11] A. De Salve, "Tapentadol PR in fragile over eighty years old patients with non-cancer chronic pain," in Proceedings of the 8th World Congress of the World Institute of Pain, Hilton, NY, USA, May 2016, http://simuleurope.com/2016/wip/Posters (poster presentation), http://onlinelibrary.wiley.com/doi/10. 1111/papr.12451/epdf.
[12] D. M. Biondi, J. Xiang, M. Etropolski, and B. Moskovitz, "Tolerability and efficacy of tapentadol extended release in elderly patients $\geq 75$ years of age with chronic osteoarthritis knee or low back pain," Journal of Opioid Management, vol. 11, no. 5, pp. 393-403, 2015.

[13] B. Lange, M. Sohns, J. Tempero, and C. Elling, "Efficacy and safety of tapentadol prolonged release formulation in the treatment of elderly patients with moderate to severe chronic osteoarthritis knee pain: a pooled analysis of two doubleblind, randomized, placebo- and active-controlled trials," Current Medical Research and Opinion, vol. 34, no. 12, pp. 2113-2123, 2018.

[14] B. Lange, D. von Zabern, C. Elling, and C. Dubois, "Efficacy and safety of tapentadol prolonged release for moderate-tosevere chronic osteoarthritis knee pain: a pooled analysis of two double-blind, randomized, placebo- and oxycodone controlled release-controlled studies," Current Medical Research and Opinion, vol. 33, pp. 1413-1422, 2017.

[15] R. B. Raffa, C. Elling, and T. M. Tzschentke, "Does 'strong analgesic' equal 'strong opioid?' Tapentadol and the concept of ' $\mu$-load'," Advances in Therapy, vol. 35, no. 10, pp. 1471-1484, 2018.

[16] T. M. Tzschentke, T. Christoph, and B. Y. Kögel, "The muopioid receptor agonist/noradrenaline reuptake inhibition (MOR-NRI) concept in analgesia: the case of tapentadol," CNS Drugs, vol. 28, pp. 319-329, 2014.

[17] A. Schwittay, C. Schumann, B. Litzenburger, and K. Schwenke, "Tapentadol prolonged release for severe chronic pain: results of a noninterventional study involving general practitioners and internists," Journal of Pain \& Palliative Care Pharmacotherapy, vol. 27, no. 3, pp. 225-234, 2013.

[18] Grünenthal GmbH, Fachinformation Palexia ${ }^{\circledR}$ Retard (German Summary of Product Characteristics), Grünenthal GmbH, Aachen, Germany, 2010.

[19] T. J. Kiresuk and R. E. Sherman, "Goal attainment scaling: a general method for evaluating comprehensive community mental health programs," Community Mental Health Journal, vol. 4, no. 6, pp. 443-453, 1968.

[20] T. Lange, D. Krings, and S. Waldmann-Rex, "Clinical practice data regarding tapentadol prolonged release treatment for severe chronic pain-improvement of analgesia, functional competence and quality of life in particular under tapentadol monotherapy (in German)," MMW-Fortschritte der Medizin, vol. 157, no. 4, pp. 12-21, 2015.

[21] P. C. Langley, "The societal burden of pain in Germany: healthrelated quality-of-life, health status and direct medical costs," Journal of Medical Economics, vol. 15, no. 6, pp. 1-15, 2012.

[22] Osteoarthritis Research Society International, Osteoarthritis: A Serious Disease, 2016, https://www.oarsi.org/sites/default/ files/docs/2016/oarsi_white_paper_oa_serious_disease_121416_1. pdf.

[23] M. Strohmeier, S. Waldmann-Rex, and K. Schwenke, "Influence of tapentadol retard on functional parameters in patients with severe chronic pain-is there a benefit in daily life?" MMW-Fortschritte der Medizin, vol. 155, pp. 63-71, 2013, in German.

[24] V. Strick, "Management of severe chronic pain with tapentadol prolonged release-long-term data from pain specialists," Current Medical Research and Opinion, vol. 30, no. 10, pp. 2085-2092, 2014.

[25] A. Schikowski, D. Krings, and K. Schwenke, "Tapentadol prolonged release for severe chronic cancer-related pain: 
effectiveness, tolerability, and influence on quality of life of the patients," Journal of Pain Research, vol. 8, pp. 1-8, 2015.

[26] K. Schwenke, A. Agbalaka, and B. Litzenburger, "Tapentadol prolonged release as used in clinical practice in patients with severe chronic tumor pain," Journal of Palliative Care \& Medicine, vol. 5, no. 2, p. 211, 2015.

[27] K. U. Kern, D. Krings, and S. Waldmann-Rex, "Tapentadol prolonged release improves analgesia, functional impairment and quality of life in patients with chronic pain who have previously received oxycodone/naloxone," Journal of Pain \& Relief, vol. 6, no. 1, p. 281, 2017. 